Apidologie, 1978, 9 (1), 57-73.

\title{
WABENORIENTIERUNG IM NEST DER HONIGBIENE (APIS MELLIFICA L.)
}

\author{
Orientation des rayons dans le nid \\ de l'abeille domestique (Apis mellifica $\mathrm{L}$.)
}

\author{
Michael D. IFANTIDIS \\ Institut für Bienenkunde* der Landwirtschaftlichen \\ Fakultät der Universität Thessaloniki, Griechenland
}

\author{
SUMMARY \\ COMB ORIENTATION IN THE HONEYBEE \\ (Apis mellifica L.) NEST
}

In the area of Thessaloniki, Greece, during the years 1974 to 1976 we had several bee colonies of Apis mellifera cecropia to build repeatedly new combs directly on the cardboard cover of two types of beehives with concave walls. According to our experiments the orientation of the combs does not depend on the magnetic field of earth, shape of the cover, shape of the interior of the beehive and position of the entrance.

\section{ZUSAMMENFASSUNG}

In der Gegend von Thessaloniki, Griechenland, liessen wir in den Jahren 1974 bis 1976 mehrere Bienenvölker von Apis mellifica cecropia in zwei Beutentypen mit gewölbten Wänden wiederholt neue Waben direkt an dem aus Karton bestehenden Deckel bauen. Nach unseren Untersuchungen haben sich Erdmagnetfeld, Form des Deckels bzw. des Hohlraumes der Beute und Lage des Fluglochs als unbedeutende Faktoren für die Wabenorientierung im Bienenvolk erwiesen.

\footnotetext{
* Die finanzielle Unterstützung der vorliegenden Arbeit übernahm das hiesige Institut für Bienenkunde.
} 


\section{EINLEITUNG}

Der Wabenbau der Honigbiene fand schon früh das Interesse der Bienenforscher, die unter anderem die Gestalt der Zellen, die parallele Anordnung der Waben, ihren Herstellungsrhythmus usw. untersuchten. R. DARCHEN (1968), der sich jahrelang mit dem Wabenbau experimentell befasste, hat die umfangreiche Bibliographie darüber zusammengestellt und vorgelegt.

St. TABER und Ch. D. Owens (1971) haben den Einfluss der Temperatur auf die Auswahl der Stelle des Wabenansatzes am Deckel der Bienenwohnung studiert. Dieselben Autoren (1973) untersuchten auch die Grösse und Gestalt der Bienenwabe, um Informationen über die Herstellung einer bienengerechten Beute zu erhalten.

Noch spärlich und verhältnismässig neu sind die Arbeiten über die Wabenrichtung bei der Honigbiene. E. Crane (1974) präsentiert in ihrem Artikel "Directions in which bees build combs " die bezügliche Bibliographie. In einer nicht veröffentlichten, von H. MARTIN und M. LINDAUER (1973) erwähnten Arbeit stellte M. OenmKe fest, dass die Orientierung der Waben vom Erdmagnetfeld beeinflusst wird, und dass die Waben eines Schwarmes so ausgerichtet sind wie im Muttervolk.

Interessant ist die Richtungsweise der Waben eines Volkes in einem liegenden Zylinder, in dem es kein Rähmchen gibt. Diese Beutenform ist uns als die urtümliche bekannt (E. Herold, 1972 a) und wird auch heute in ihrer Heimat, nämlich in Ägypten (B. ADAM, 1965) wie auch in anderen Ländern benutzt. In Zypern gibt es sie ebenfalls (A. J. Gracham, 1975) und zwar mit dem Namen."Ziwerti ”(K. Miltiadis, 1956). In Beuten dieser Form zeigt sich die verschiedenartige Orientierung der Waben bei den einzelnen Völkern besonders gut. Die zypriotischen Imker unterteilen sogar die in dem "Ziwerti » untergebrachten Bienenvölker auf Grund der Wabenrichtung in drei Gruppen, nämlich in : a) "koskinades "*, mit Waben senkrecht zur Längsachse des Zylinders, $b$ ) " ochsites " und $c$ ) " spathades " mit parallelen bzw. schrägen Waben zur selben Achse (K. Miltiadis, 1956).

Die erläuterte Situation in Zypern war uns Anlass, die möglichen Umweltfaktoren zu erforschen, welche die Wabenorientierung im Nest der Honigbiene beeinflussen. Die Versuche wurden 1974 bis 1976 in Thessaloniki durchgeführt.

\section{MATERIAL UND METHODEN}

\section{1. - Die Beuten}

Zwei verschiedene Beutentypen wurden in unseren Experimenten verwendet. Die eine Beute bestand aus Lehm und hatte die Form eines umgekippten Kegelstumpfes (Blumentopf mit losem Boden). Die Durchmesser ihrer Grundflächen betrugen 0,30 bzw. $0,12 \mathrm{~m}$, die Länge ihrer Seitenlinie $0,30 \mathrm{~m}$. Der Boden war nicht fest mit dem Beutenkörper verbunden. Er hatte in der Mitte ein rundes Flugloch von etwa

* "Koskino wheisst auf deutsch das Sieb, "ochsis » spitzig und " spathi ” der Säbel. 
$0,02 \mathrm{~m}$ Durchmesser, das bei einem Teil der Versuche fehlte. Ein zweites Loch mit einem Durchmesser von $0,05 \mathrm{~m}$ befand sich in der Wand, $0,10 \mathrm{~m}$ vom Deckel entfernt. Dieses Flugloch wurde abwechselnd mit dem ersten benutzt. Ein viereckiges Fensterchen aus durchsichtigem Plexiglas $(0,10 \times 0,10 \mathrm{~m})$ befand sich in gleicher Höhe wie das seitliche Flugloch. Durch dieses Fensterchen liessen wir Licht in die Beute eindringen, um seine Wirkung auf die Wabenorientierung zu untersuchen. In diesem Falle wurde das Flugloch im Boden verwendet. Das Licht blieb wochenlang ununterbrochen Tag und Nacht mit Hilfe eines Scheinwerfers von $150 \mathrm{~W}$ erhalten. Die Lichtquelle wurde $2 \mathrm{~m}$ vom Fensterchen entfernt aufgestellt.

Der zweite Beutentyp bestand aus Zement. Der Hohlraum war ein Ellipsoid mit horizontalen Achsenlängen von 0,40 bzw. $0,20 \mathrm{~m}$. Dieser Raum wurde etwas verkleinert, indem seine obere Wölbung entfernt wurde. Auf diese Weise betrug die endgültige Höhe $0,17 \mathrm{~m}$. Das Flugloch befand sich am niedrigsten Punkt des Hohlraumes. Äusserlich hatte diese Beute die Form eines Quaders in den Dimensionen $0,50 \times 0,30 \times 0,20 \mathrm{~m}$ (Abb. $6 \mathrm{a}$, letztes Bild).

Unabhängig vom Beutentyp bestand der Deckel, an dem die Waben angesetzt wurden, aus Karton. Im Abstand von einem bis drei (selten bis vier) Tagen wurde der Deckel ersetzt, so dass die Bienen immer wieder neue Waben bauen mussten.

Bei einem Versuch wurde der Raum der ellipsoidischen Beute mit einem Streifen Karton oder biegsamem Kunststoff von 0,04 m Breite und $1 \mathrm{~mm}$ Dicke zweigeteilt. Zuerst wurde dieser "Kragen " in die Beute selbst (Abb. 6 a), danach aber auf den Deckel geklebt (Abb. 6 c u. d).

\section{2. - Die Bienen}

Insgesamt wurden in unseren Versuchen 13 Völker der Honigbiene Apis mellifica cecropia verwendet. Die Bienenschwärme waren gross genug (ca $1500 \mathrm{~g}$ ), um den verfügbaren Hohlraum ganz zu füllen. Das hatte zur Folge, dass die Bienentraube zwangsläufig die Form des jeweiligen Raums annahm. Die Schwärme wurden aus Körben abgetrommelt, nachdem die Richtung ihrer Waben zur Kompassrichtung registriert worden war.

Ein Flügel der Königin jedes Volkes wurde gestutzt, damit der Schwarm, auch unter den biologisch ungünstigen Versuchsbedingungen (keine Wabe, keine Brut, kein Futtervorrat), seine Wohnung möglichst nicht verliess. Es lag mir daran, die Königin nicht zu verlieren, denn sie hat eine direkte Wirkung auf Art und Dauer des Wabenbaues (R. DARCHEN, 1968).

\section{3. - Die Fütterung}

Die Fütterung erfolgte mit Zuckerteig oder mit Zuckerlösung. Die Futtergefässe wurden entweder in der Nähe der Beute (Abb. 6 a) oder, in fortgeschrittener Jahreszeit, auf deren Boden aufgestellt.

\section{4. - Die Messung der Winkelabweichung}

Die Abweichung der Wabenrichtung von der Richtung des jeweilig geprüften Faktors wurde durch den ersten Winkel gemessen, der links von der Richtungslinie dieses Faktors gebildet wurde. Die Richtung des Erdmagnetfeldes wurde auf die Oberfläche des Deckels gezeichnet. Nach dem Wechsel des Deckels wurde sie auf die innere Seite des Kartons übertragen, indem wir die aufgezeichnete Linie mit einer Nadel an zwei Punkten durchbohrten.

\section{ERGEBNISSE}

Die Ergebnisse über die Bedeutung des Faktors "Erdmagnetfeld" werden in Tabelle 1 und in Abbildung 1 dargestellt.

Die Daten der Tabelle 2 und die Fotos der Abbildung 2 beziehen sich auf den Faktor "Form des Deckels bzw. des Hohlraumes". 


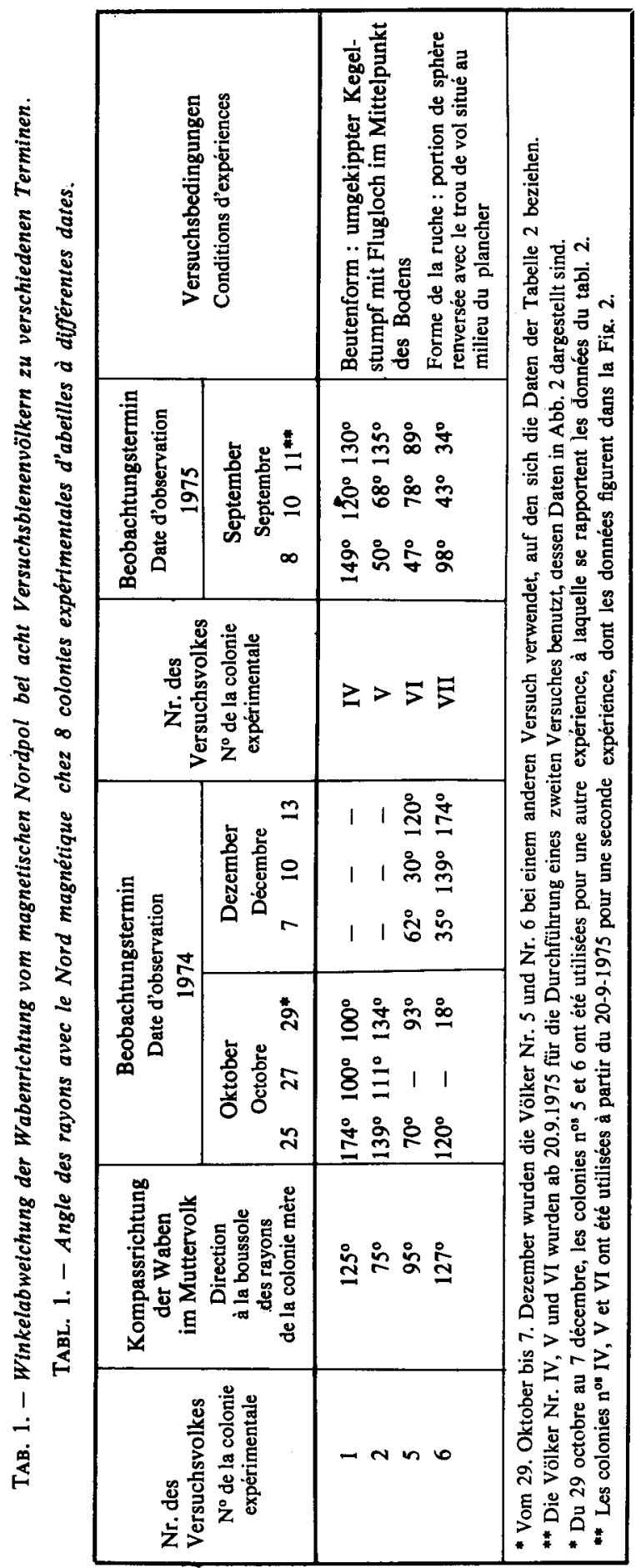




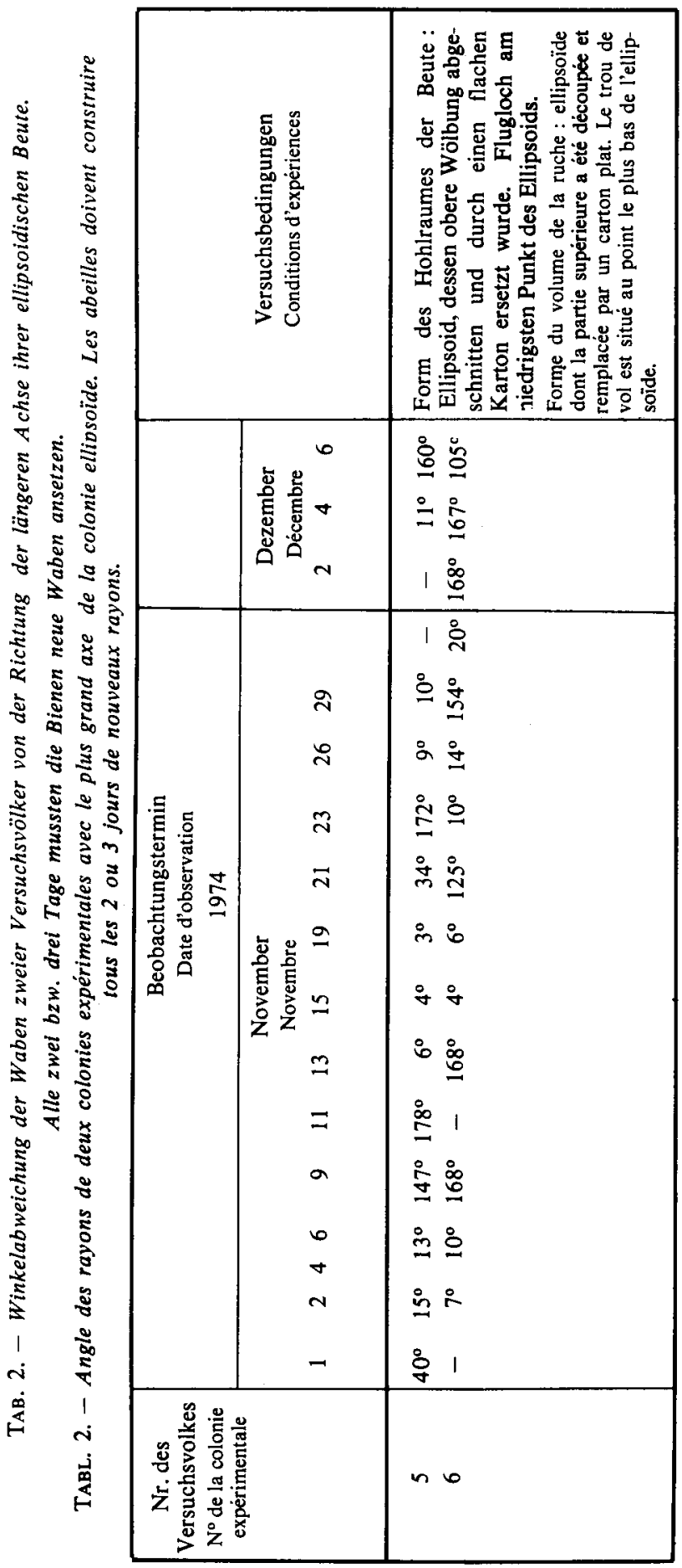




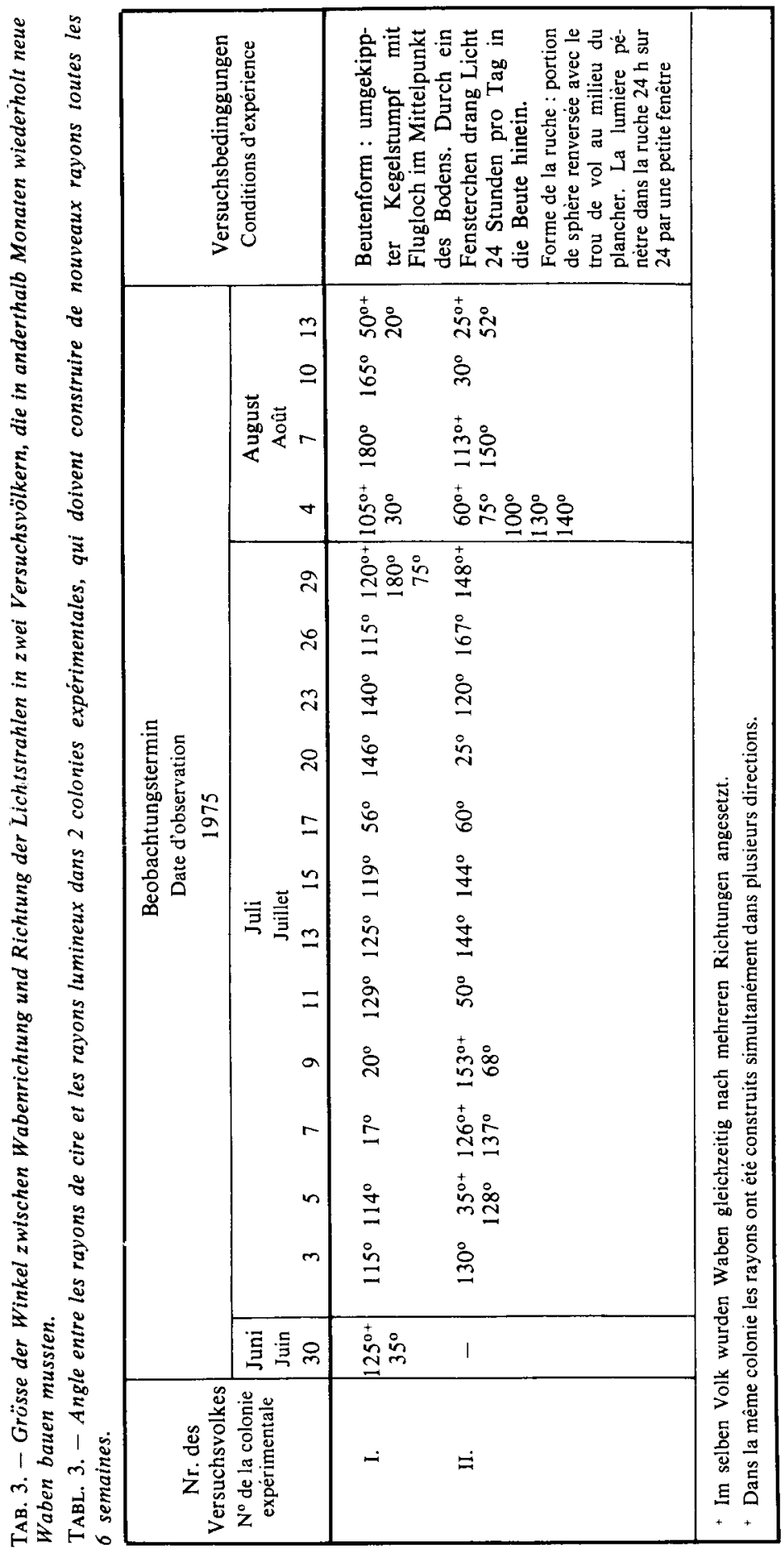



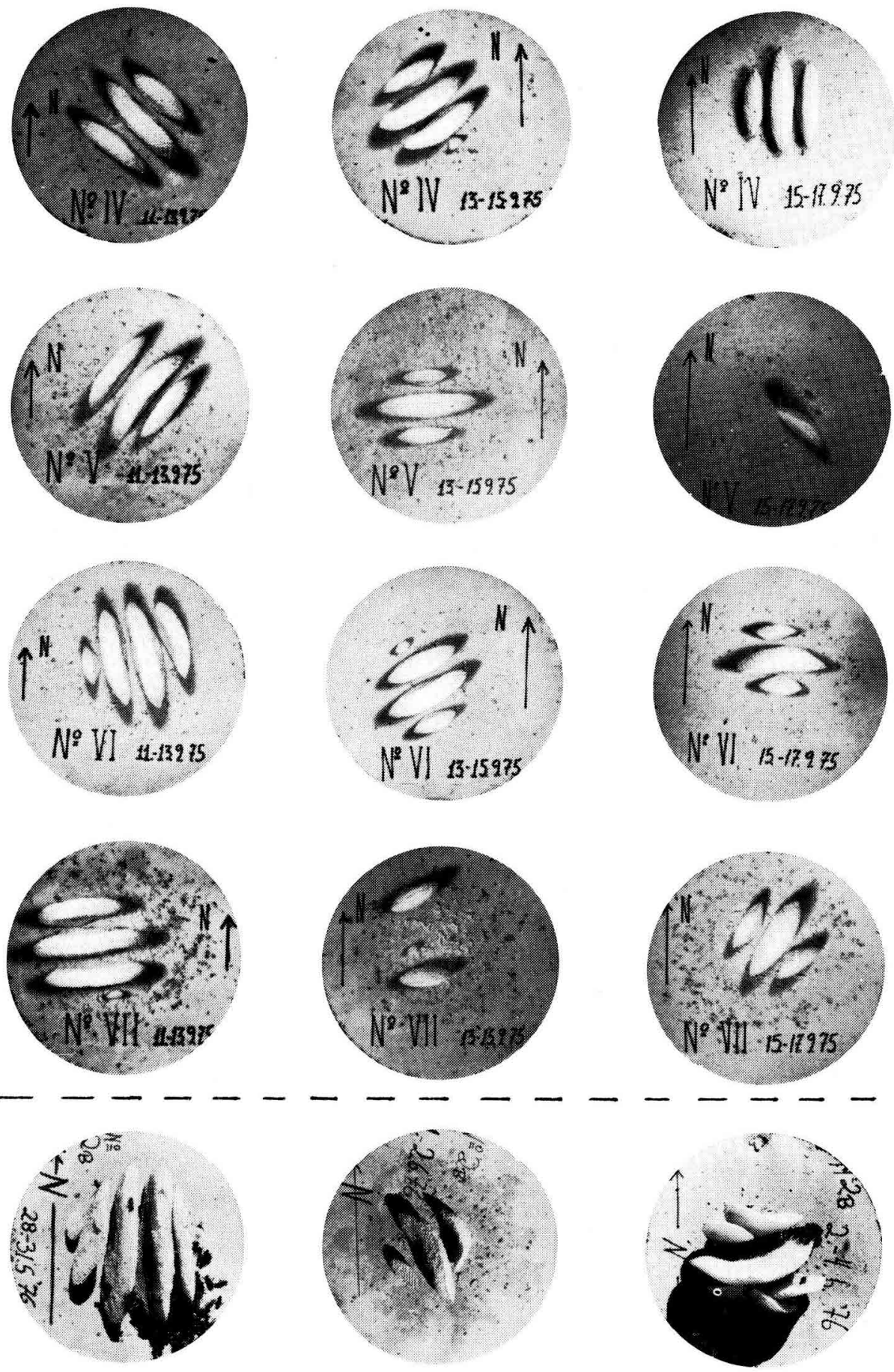

Авв. 1. - A ufnahmen von Bienenwaben, die von fünf Versuchsvölkern (Nr. IV, V, VI, VII und 2 B)

im Jahre 1975 (a) und 1976 (b) wiederholt und direkt an den Deckel gebaut wurden.

Die Beute hatte die Form eines umgekippten Kegelstumpfes, ihr Flugloch war im Mittelpunkt des Bodens. Jede einzelne Aufnahme wurde so ausgerichtet, dass der Pfeil, der die Richtung des magnetischen Erdpols angibt, senkrecht nach oben zeigt.

FIG. 1. - Photographie des rayons que 5 colonies expérimentales ( $N^{o} I V, V, V I, V I I$ et 2 B) ont construits à plusieurs reprises en 1975 (a) et 1976 (b) directement au plafond.

La ruche avait la forme d'une portion de sphère renversée, son trou de vol était situé au milieu du plancher. Chaque photographie a été redressée, de sorte que la flèche,

qui indique la direction du Nord magnétique, soit dirigée verticalement vers le haut. 

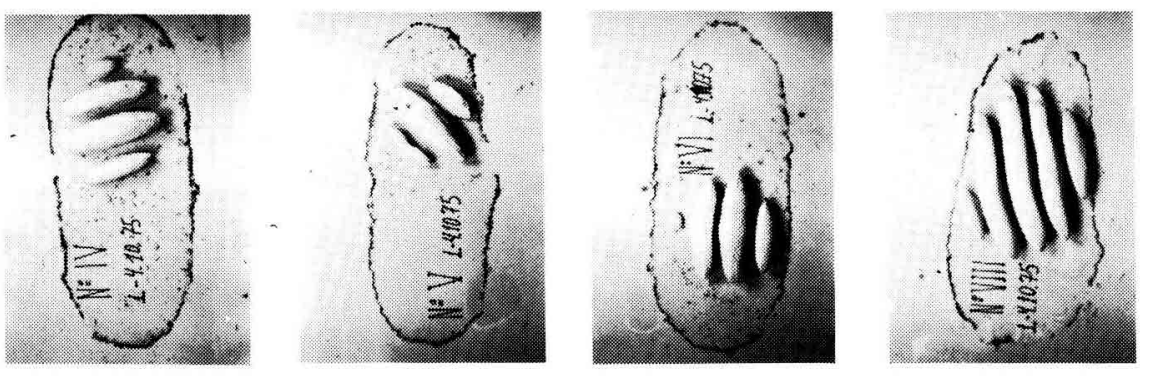

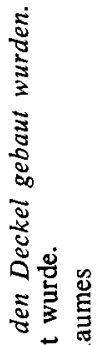

ํํㄹ
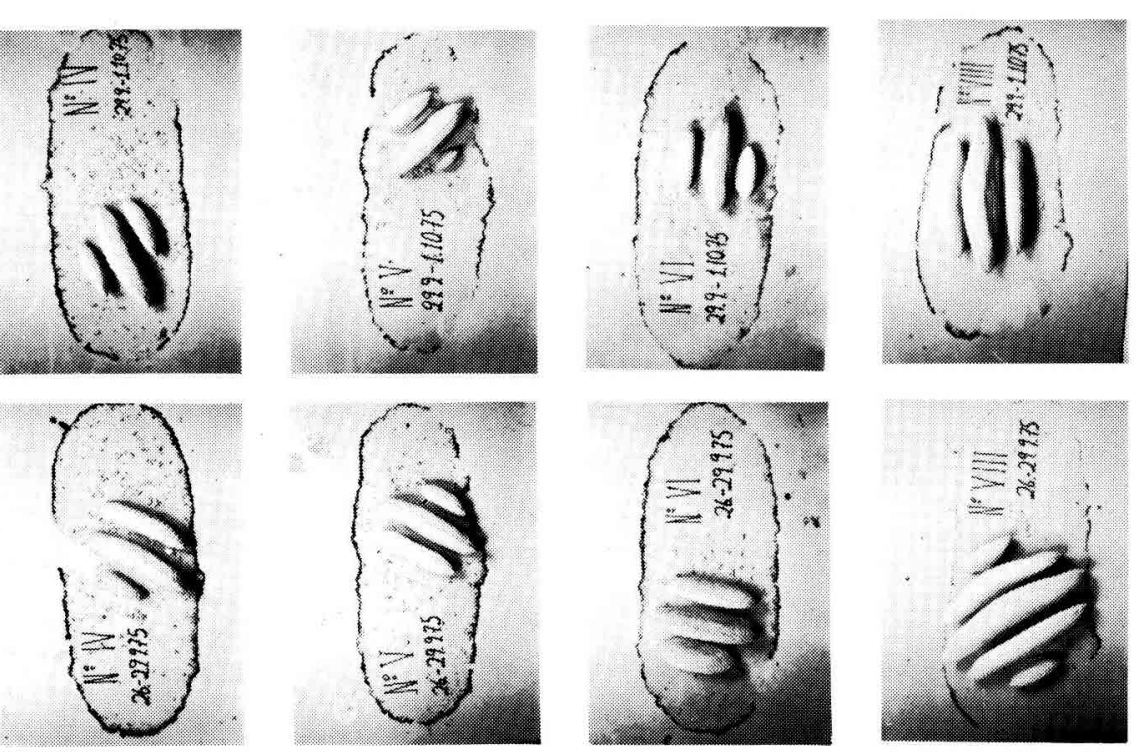

ธง 요

可要

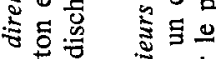

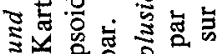

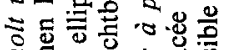

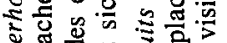

速

ลิ.

둥영

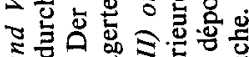

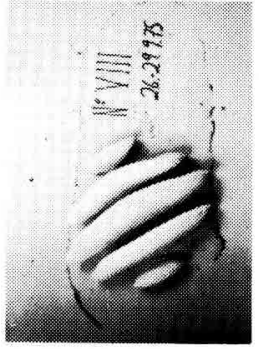

or os $\mathrm{s}$

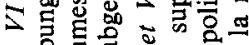

入

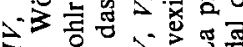

원영

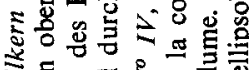

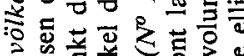

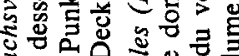

行

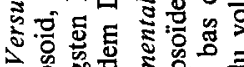

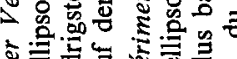

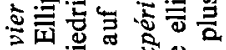

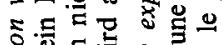

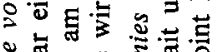

范范势

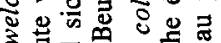

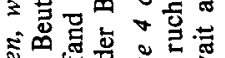
ปิ๊

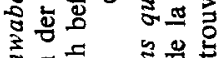

ปี

今ั

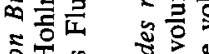

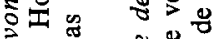

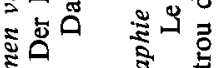

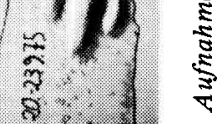

क्ष

301

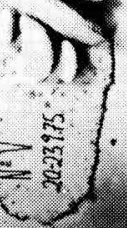

1) $\frac{2}{9}$

넌, 1

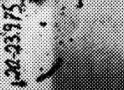

1,

N 

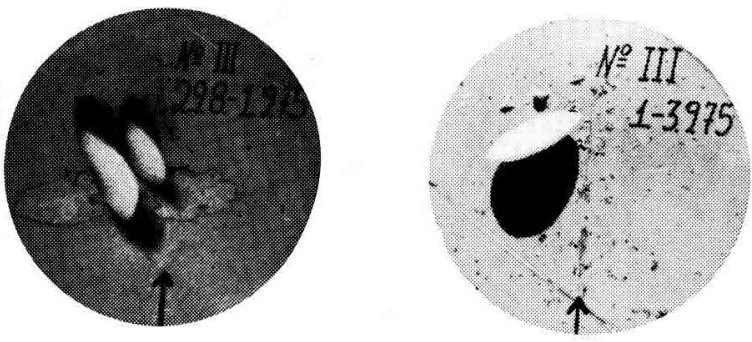

\section{das Volk hat die Beute verlassen}
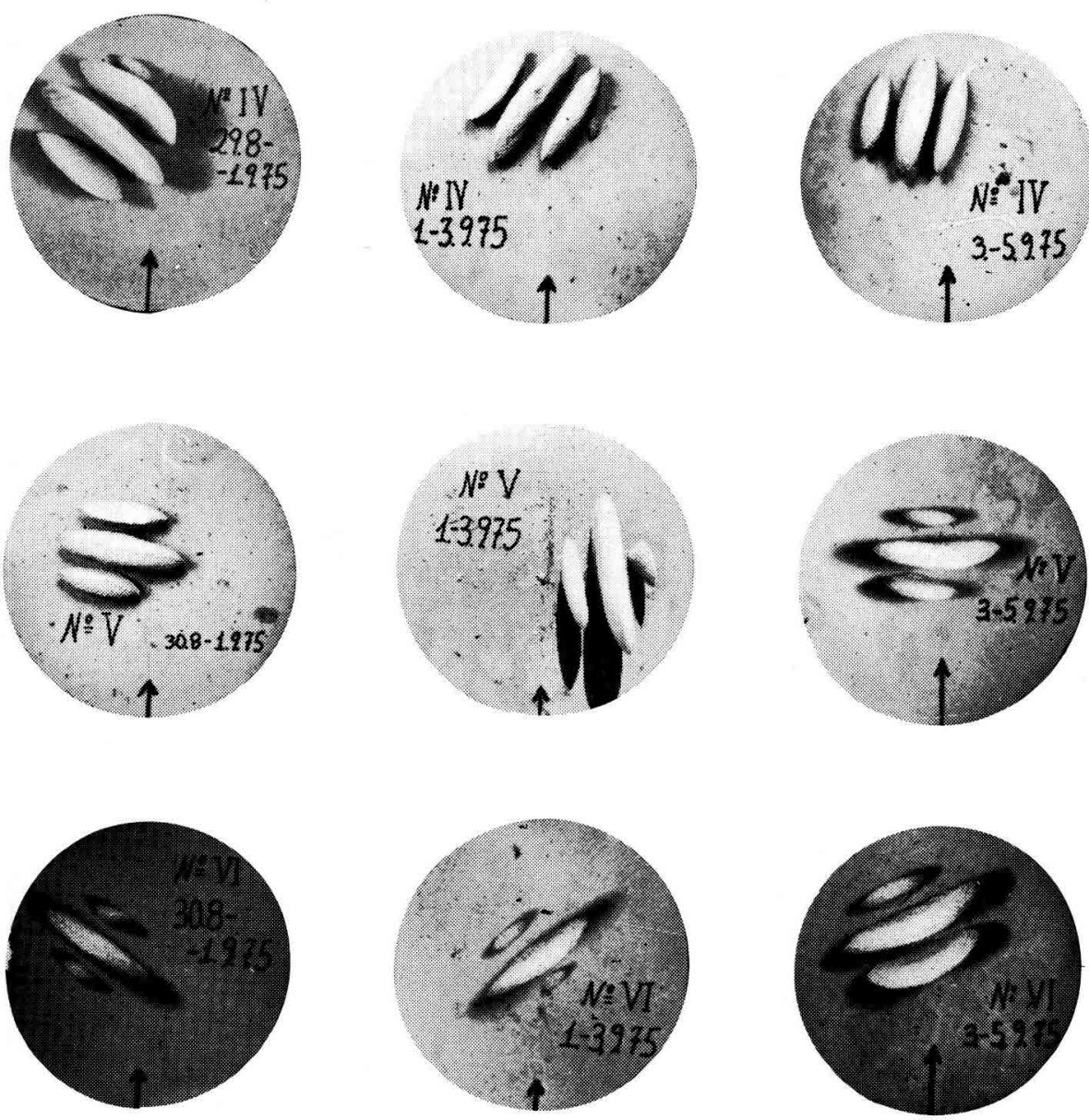

Авв. 3. - Fotos von Waben, die von vier Versuchsvölkern (Nr. III, IV, V und VI) in einem Blumentopf mit dem Flugloch in der Wand wiederholt gebaut wurden. Jede Aufnahme wurde so orientiert, dass der Pfeil,

der die Einflugrichtung der Bienen in die Beute anzeigt, senkrecht nach oben gerichtet ist.

FIG. 3. - Photographies de rayons, que 4 colonies expérimentales ( $N^{\circ} \mathrm{III}, I V, V$ et VI) ont construits à plusieurs reprises dans un pot de fleurs avec le trou de vol sur la paroi.

Chaque cliché a été orienté de sorte que la flèche, qui indique la direction de vol des abeilles pour entrer dans la ruche, soit dirigée verticalement vers le haut. 

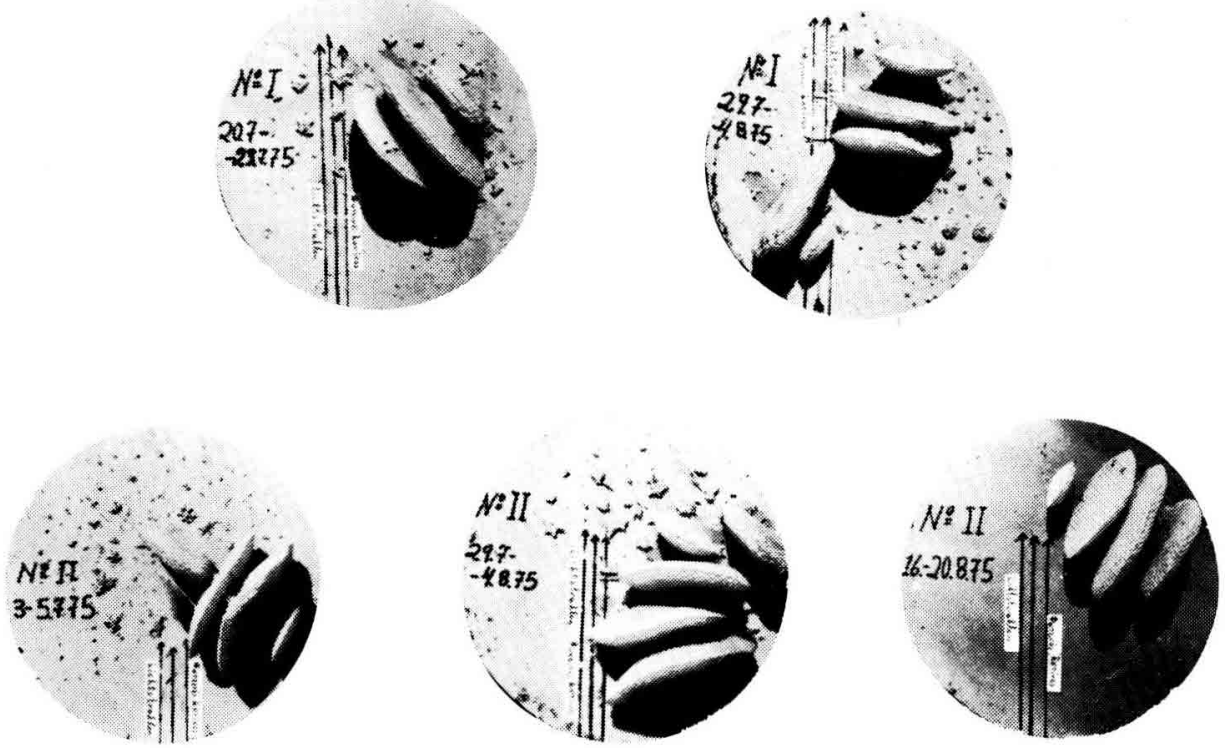

Авв. 4. - Einige Fotos (Völker Nr. 1 und II) aus dem Versuch über den Einfluss ständigen Lichtes auf die Orientierung der Bienenwaben. (Ausführlichere Daten in Tabelle 3).

Die parallelen Linien auf den Fotos stellen die Richtung der Lichtstrahlen dar.

FIG. 4. - Quelques photographies (colonies $n^{o} I$ et II) concernant l'expérience sur l'influence de la lumière constante sur l'orientation des rayons

(données détaillées dans le tableau 3).

Les lignes parallèles sur les photos représentent la direction des rayons lumineux,
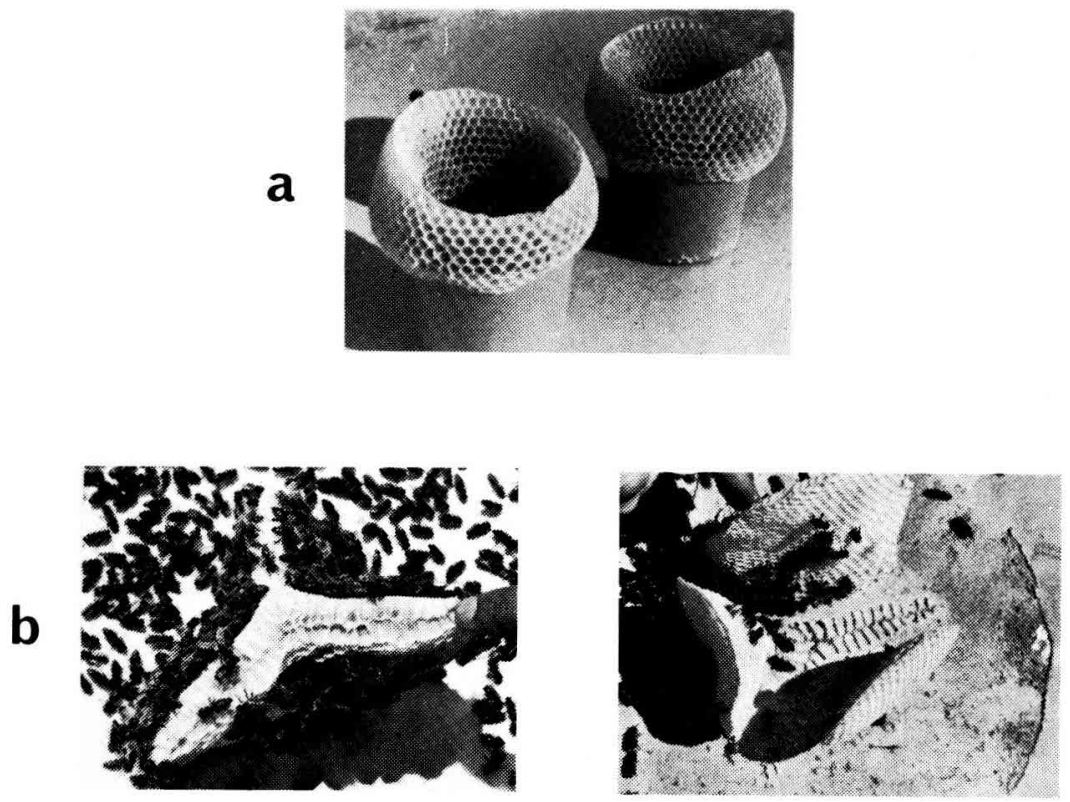

AвB. 5. - Waben ungewöhnlicher Gestalt von Apis mellifica. Erläuterungen im Text im Abschnitt Ergebnisse.

FIG. 5. - Rayons de forme inhabituelle d'Apis mellifica. Explications dans le résumé long au paragraphe Résultats. 

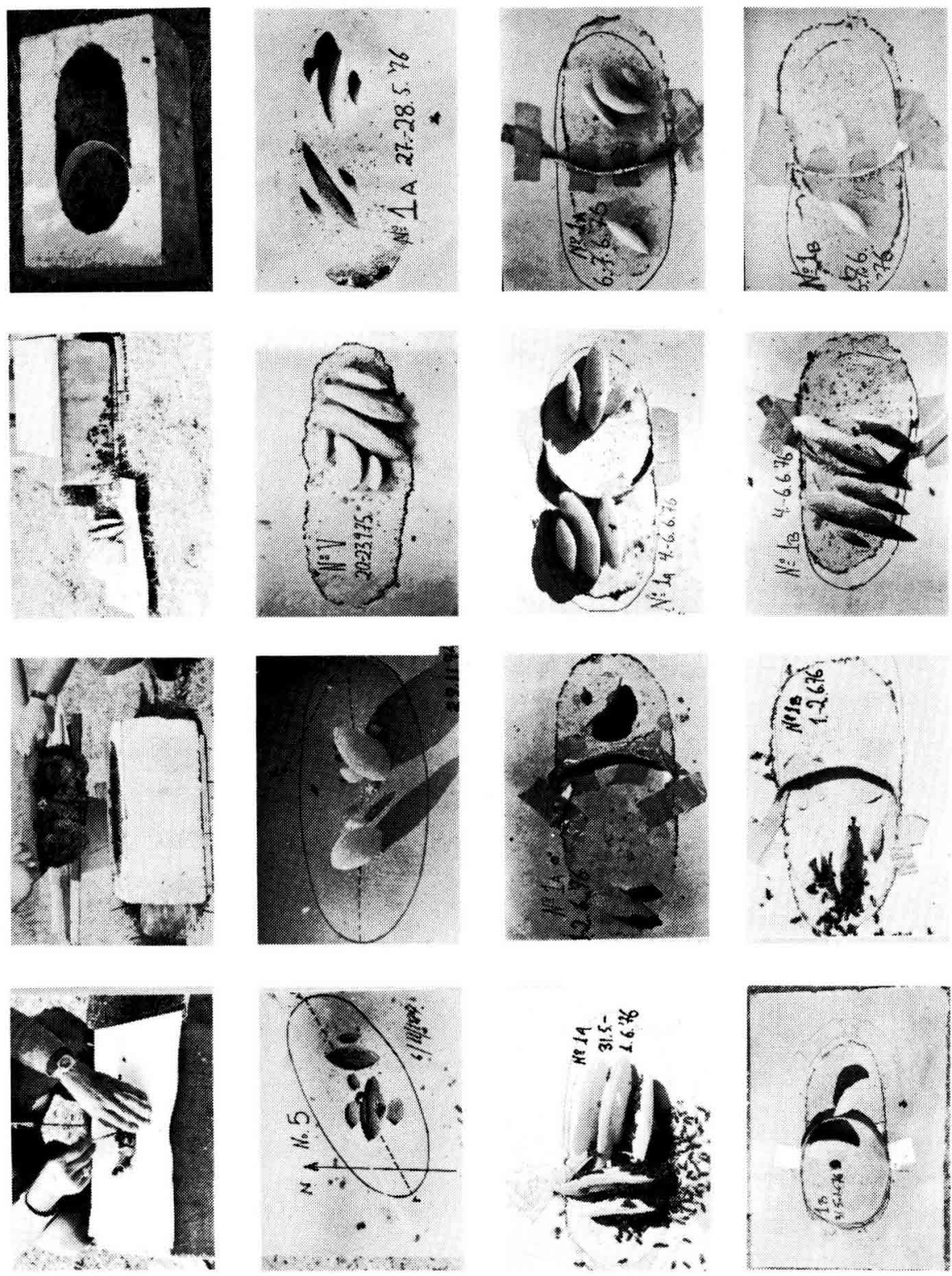

๘

$\cup$

Aвв. 6. - a) Arbeitsstufen beim Wechsel des Deckels der Beute mit ellipsoidischem Hohlraum. Das letzte Foto zeigt sowohl die äussere Form der Beute wie auch die Form des inneren Raumes, mit dem Flugloch an seinem niedrigsten Punkt.

Man sieht auch den "Kragen " für die partielle Trennung der Bienentraube.

b) Auswahl einiger Fälle, bei denen die Versuchsvölker ihre Waben nicht nur an zwei getrennten Stellen des Deckels sondern auch nach verschiedenen Richtungen gebaut haben.

c u. d) Absichtliches Verursachen des in Abb. 6 b dargestellten Phänomens mit Hilfe des "Kragens».

FIG. 6. - a) Étapes du travail lors du changement du toit de la ruche à volume ellipsoïdal.

La dernière photo montre à la fois la forme extérieure de la ruche et la forme du volume intérieur, avec le trou de vol situé au point le plus bas.

On voit également le "col " pour séparer partiellement la grappe d'abeilles.

b) Sélection de quelques cas dans lesquels les abeilles ont construit leurs rayons non seulement en deux endroits différents du plafond, mais aussi dans diverses directions.

$\mathrm{c}$ et d) Production intentionnelle du phénomène représenté à la Fig. 6 bà l'aide d'un a col ». 
Tabelle 3 betrifft den Einfluss des Lichtes auf die Orientierung der Waben, Abbildung 4 bringt einige Fälle aus Tabelle 3 in Form von Fotos.

Für den Faktor "Lage des Flugloches " haben wir Daten nur als Fotoaufnahmen (Abb. 3).

Abbildung 5 a zeigt den unerwarteten Fall, dass die Bienen ihre Waben nicht nur von unten nach oben, sondern auch in Form eines Zylinders bauten. Das Phänomen kam bei zwei Versuchsvölkern vor, die bis Mitte Dezember gezwungen waren, neue Waben zu bauen. Wegen der niedrigen Temperaturen zu dieser Jahreszeit mussten die Bienen bei dem Futtergefäss verbleiben, das sich auf dem Boden der Beute befand. Auf diese Weise stellten sie ihre Versorgung mit Zuckerteig sicher.

In der Abbildung $5 \mathrm{~b}$ wird der ebenfalls ungewöhnliche Fall gezeigt, dass die Bienen eine Wabe bauten, welche im Querschnitt die Form eines Ypsilons (Y) aufweist (erste Aufnahme). Die zweite Aufnahme derselben Abbildung zeigt die Spuren der absichtlich entfernten ypsilonförmigen Wabe und die anderen drei Waben, die in dreieckiger Anordnung um sie herum angesetzt worden waren.

\section{DISKUSSION}

Bekanntlich lässt sich die Richtung jeder Wabe eines Bienenvolkes durch eine Leiste (Rähmchen) oder durch eine senkrechte Fläche (Beutenwand, andere Wabe) leicht festlegen. Aus diesem Grund hatten alle Versuchsbeuten nur gewölbte Wände, und die Bienen mussten ihre Waben direkt an den Deckel bauen, der aus flachem Karton bestand.

In solchen Beuten könnten vielleicht folgende Faktoren die Wabenorientierung beeinflussen : a) das Erdmagnetfeld, weil die Bienen es wahrnehmen (M. LINDAUER und H. MARTIN, 1968), b) die Form des Deckels bzw. des Hohlraumes, unter der Bedingung, dass die Bienenmasse gross genug ist, um den ganzen verfügbaren Raum zu füllen und $c$ ) die Lage des Fluglochs (einseitiger Zustrom von Licht und Luft).

\section{Erdmagnetfeld}

In einer Beute mit rundem Deckel und mit dem Flugloch im Mittelpunkt des Bodens bleibt als einziger Faktor von den oben erwähnten das Erdmagnetfeld. Wie aus den Daten der Tabelle 1 bzw. aus den Fotos der Abbildung 1 ersichtlich wird, haben die Bienen ihre Waben nach ganz zufälligen Richtungen gebaut, d.h. unbeeinflusst vom Erdmagnetfeld. Die Wabenorientierung war verschieden, nicht nur in den einzelnen Versuchsvölkern am selben Tag, sondern auch in jedem einzelnen Volk in den nacheinander folgenden Zeitabschnitten. Unsere Feststellung, dass das irdische Magnetfeld die Richtung der Bienenwabe nicht beeinflusst, steht derjenigen von OehmKe (Martin und Lindauer, 1973) gegenüber. Ob dafür der unterschiedliche Wert der Magnetintensität der Erde zwischen Griechenland (0,3 Gauss : Alexopoulos, 1959) und Deutschland (0,5 Gauss : Martin und Lindauer, 1973) 
verantwortlich ist, kann durch unsere Experimente nicht entschieden werden. Wir konnten auch den Befund des genannten Autors nicht bestätigen, dass die ersten Waben eines Bienenschwarmes in einem Kartonzylinder so ausgerichtet sind wie im Muttervolk. Das wird aus dem Vergleich zwischen den Kolumnen zwei und drei der Tabelle 1 ersichtlich.

\section{Lage des Fluglochs}

Nachdem wir festgestellt hatten, dass das Erdmagnetfeld in der geographischen Breite von Thessaloniki die Bienen bei der Orientierung ihrer Waben nicht beeinflusst, haben wir in der gleichen Beutenform (Blumentopf) die Wirkung der Lage des Fluglochs studiert.

Durch ein seitliches Flugloch können zwei Faktoren, nämlich Licht und Luft, gleichzeitig einwirken. Wir mussten also einen von ihnen eliminieren.

\section{a) Licht}

Zunächst blieb das Flugloch am Boden in Betrieb, während durch das seitliche Fensterchen nur Licht in die Beute eindrang. Nach R. MoRse (1965) hat die Lichtintensität eine entscheidende Wirkung auf den Beginn des Wabenbaues. Derselbe Autor fand ferner, dass ein Bienenschwarm auch nach mehr als einem Monat nicht mit dem Wabenbau anfängt, wenn er sich unter ununterbrochenem Lichteinfluss befindet. Es genügte aber eine Dunkelperiode für das Bienenvolk, um mit dem Bau von Waben in seiner neuen Wohnung zu beginnen. Danach ging der Bau unabhängig von der Lichtdauer weiter. G. TARANOv (1959) hat zudem festgestellt, dass einige Bienenschwärme bei Tage, andere aber bei Nacht Waben errichten.

Auf Grund dieser Befunde liessen wir die Versuchsvölker ihre ersten Waben unter normalen Lichtbedingungen bauen. Danach hatten sie neue Waben wiederholt und für mehrere Wochen unter der einseitigen Wirkung ständigen Lichtes zu bauen. Aus den Daten der Tabelle 3 lässt sich schliessen, dass die Richtung des Lichtes die Wabenorientierung nicht beeinflusst. Nur das Vorkommen von Wirrbau wurde etwas häufiger beobachtet (keine statistische Bearbeitung!).

\section{b) Zusammenwirken von Licht und Luft}

Beim nächsten Versuch drangen Luft und Sonnenlicht gleichzeitig durch das seitliche Flugloch in die kegelstumpfförmige Beute ein. (Das Fensterchen war völlig mit undurchsichtigem Stoff bedeckt). Abbildung 3 macht deutlich, dass auch der Faktor " Lage des Fluglochs " keinen Einfluss auf die Wabenrichtung hat, weil sie von Volk zu Volk und von einem Zeitpunkt zum anderen beim gleichen Volk verschieden war.

Form des Deckels bzw. Hohlraumes

Was die Form des Deckels bzw. des Hohlraumes der Beute betrifft, so zeigen die Daten der Tabelle 2, dass die Bienen auch von ihr während des Wabenbaues nicht beeinflusst werden. Die Aufnahmen der Abbildung 2 veranschaulichen diese Tatsache. 


\section{Eine Versuchserweiterung}

Im Rahmen dieser Untersuchung wurde zufällig noch folgendes festgestellt : Es gab einige Fälle, in denen die Bienen ein und desselben Volkes ihre Waben an zwei getrennten Stellen des Deckels bauten, und zwar gleichzeitig nach verschiedenen Richtungen (Abb. 6 b). Diese Feststellung gab uns den Anlass, unsere Versuche zu erweitern.

Zuerst sei aber noch erwähnt, dass das Vorkommen von multiplen Bauzentren schon TABER und OwENS (1970) festgestellt haben, ohne jedoch klarzumachen, ob die Orientierung der Waben in den einzelnen Bauzentren die gleiche war oder nicht.

Dürfen wir nun diese Fälle ohne weiteres als einen zusätzlichen Beweis dafür ansehen, dass die Bienen eines Volkes bei der Wabenorientierung, zumindest von den hier geprüften Umweltfaktoren, nicht beeinflusst werden? Oder war das Vorkommen von zwei Bauzentren mit den zueinander nicht parallelen Wabengruppen das Ergebnis irgendeines unbemerkten Versuchsfehlers? Wenn das letztere nicht zutrifft, so müsste diese Situation reproduzierbar sein. Um dies nachzuprüfen, benutzten wir die Methode des “ Kragens ” (siehe S. 59). Auf diese Weise bleibt zunächst die Kommunikationsmöglichkeit zwischen den Bienen des Volkes wie auch ihr mechanischer Zusammenhang erhalten. Ausserdem wird das Vorkommen von senkrechten Flächen in dem Beutenraum durch die Anwendung des "Kragens » systematisch vermieden; parallel dazu wird durch diese Barriere das Bauen einer die ganze Länge des Deckels durchlaufenden Wabe nicht gestattet.

Wenn nun die Bienen eines Volkes bei der Orientierung ihrer Waben zu einem gewissen Zeitpunkt durch irgendeinen Faktor (z.B. Erdmagnetfeld) beeinflusst werden, dann müssen sich die Bienen der zwei Bauzentren, welche durch die neue Versuchsanordnung fast immer entstehen, koordinieren und ihre Waben ständig nach der gleichen Richtung bauen. Die in Abbildung $6 \mathrm{c}$ u. d dargestellten Ergebnisse machen aber deutlich, dass von einem solchen Koordinieren keine Rede sein kann. Die Gruppen jedes Versuchsvolkes haben ihre Waben entweder nach der selben oder nach verschiedenen Richtungen gebaut, unabhängig davon, ob sie um den " Kragen " herum kompakt blieben (Nr. 1 A, 31.5.-1.6.1976 und Nr. 1 B, 4.-6.6.1976), oder ob sie an zwei voneinander entfernten Stellen hingen (Nr. 1 A, 1.-2.6.1976 und 1 A, 4.-6.6.1976). Die Ergebnisse dieses Versuches dürften der endgültige Beweis dafür sein, dass die Bienen ihre Waben nach beliebigen Richtungen bauen.

\section{Waben von ungewöhnlicher Gestalt}

Zylinderförmige Waben werden in der Literatur schon erwähnt : T.S. K. und M. P. Johansson (1968) berichten in ihrem Artikel "Circular comb sections " über Arbeiten, die verschiedene technische Massnahmen für den Wabenbau auf zylindrisch geformten Mittelwänden betreffen. H. MarTiN und M. LiNDAUER (1973) zeigen eine Zylinderwabe, welche in einem 10 fach verstärkten, radiär verzerrten Magnetfeld angelegt wurde. 
Der in der Abbildung 5 a dargestellte Fall, bei dem zwei Bienenvölker je eine Zylinderwabe bauten (siehe Seite 68), könnte eine Bestätigung dafür sein, dass das Relief der Grundfläche, auf der die Waben angesetzt werden, der entscheidende Faktor für die Wabenrichtung ist. Dies wäre von grosser Bedeutung für die Bienen selbst, weil sie dadurch die in der Natur gebotenen Hohlräume mit ihren unebenen Decken ganz unabhängig von ihrer Form und Orientierung ausnutzen und so ihre Überebenschancen steigern könnten.

\section{DANK}

Meinen Dank möchte ich Herrn Diplomlandwirt A. Thrassyvoulou wie auch Herrn A. XANThopoulos für ihre Hilfe bei der Durchführung der Experimente ausdrücken, desgleichen Herrn Dr. St. Mariadis für seine mir wertvollen sprachlichen Hinweise.

\section{LITER ATUR VERZEICHNIS}

ADAM B., 1965. - Auf der Suche nach den besten Bienenstämmen. Südwestdeutscher Imker, 17 (1), 14-17.

Alexopoulos K. D., 1959. - Allgemeine Physik, Band Il, A. Papasissis Verlag, Athen.

Crane E., 1974. - Directions in which bees build combs. Bee World, 55 (4), 153-155.

Darchen R., 1968. - Le travail de la cire et la construction dans la ruche. Traité de biologie de l'abeille, Band II, 241-331, Masson et Cie Paris.

Herold E., 1972 a. - Neue Imkerschule, Ehrenwirth Verlag, München.

Gracham A. J., 1975. - Beehives from ancient Greece. Bee World, 56 (2), 64-75.

Johansson T. S. K. und M. P., 1968. - Circular comb sections. Bee World, 49 (4), 176-177.

Lindauer M. und Martin H., 1968. - Die Schwereorientierung der Bienen unter dem Einfluss des Erdmagnetfeldes. Z. vergl. Physiol, 60, 219-243.

Martin H. und Lindauer M., 1973. - Orientierung im Erdmagnetfeld. Fortschritte der Zoologie, 21 $(2 / 3), 211-228$.

Miltiadis K., 1956. - Abtrommeln vom zypriotischen " Tziwerti » in die moderne Beute. Melissokomiki Hellas, 6 (12), 355-358.

MORSE R. A., 1965. - The effect of light on comb construction by honeybees. Journal of Apicultural Research, 4 (1), 23-29.

OWens C. D., and TABer St. III, 1973. - Size and Shape of Comb Constructed by Honey Bee in a 1.2. $\mathrm{m}^{3}$ Box During One Season. Journal of Economic Entomology, 66 (5), 23-29.

TABer St. III and OWEns C. D., 1970. - Colony Founding and Initial Nest Desing of Honey Bees Apis Mellifera L. Animal Behav., 18, 625-632.

TABER St. III and OWENS C. D., 1971. - Influence of Temperature on Location of Clustering and Comb Building of Honey Bees. Annals of the Entomological Society of America, 64 (4), 960-961.

Taranov G. F., 1959. - The production of wax in the honey bee colony. Bee World, 40, 113-121. 


\section{RÉSUMÉ}

L'orientation variable des rayons d'une colonie d'abeilles, lorsqu'elle construit son nid dans un cylindre allongé nous a donné l'occasion d'étudier l'action possible de divers facteurs du milieu sur l'orientation des rayons chez l'Abeille domestique (Apis mellifica cecropia).

Des ruches de cette forme sont encore aujourd'hui utilisées dans divers pays, dont Chypre où on les nomme "Ziwerti » (B. AdAM, 1965; A. J. Bracham, 1975; K. Miltiadis, 1956). L'apiculteur chypriote classe les colonies d'abeilles élevées dans les "Ziwerti " en 3 groupes, selon l'orientation des rayons : a) « koskinades " dont les rayons sont perpendiculaires au plus grand axe du cylindre, b) “ ochsites » et c) " spathades " dont les rayons sont parallèles au même axe ou obliques (K. MiLtiadis, 1956).

Deux types de ruches ont été utilisés au cours des expériences menées à Thessalonique de 1974 à 1976. Tous deux avaient des parois arrondies et un toit en carton, auquel étaient fixés les rayons. Le toit était remplacé à intervalles réguliers, de 1 à 3 jours, de sorte que les abeilles avaient toujours de nouveaux rayons à construire.

L'un des types de ruche avait la forme d'un tronc de cône renversé (pot de fleurs) avec un plancher libre qui possédait un trou de vol circulaire en son milieu.

Le volume intérieur de la $2^{e}$ ruche était un ellipsoïde, dont la partie supérieure était enlevée. Le trou de vol se situait au point le plus bas de l'ellipsoïde. Extérieurement cette ruche avait la forme d'une pierre de taille (fig. 6 a, dernière vue).

Lors d'une expérience on a séparé en deux le volume intérieur du $2^{\mathrm{e}}$ type de ruche à l'aide d'un " faux col ". Il s'agissait d'une bande de carton ou de plastique souple de $0,04 \mathrm{~m}$ de large et de $1 \mathrm{~mm}$ d'épaisseur.

Les essaims (au total 13 colonies) étaient suffisamment gros pour emplir complètement le volume disponible. Il s'ensuivait que la grappe d'abeilles prenait automatiquement la forme de l'espace où elle se trouvait. Après avoir noté la direction des rayons par rapport au nord magnétique, on chassait les essaims hors des ruches.

Dans chaque colonie on a clippé une aile de la reine pour que l'essaim, même dans des conditions d'expériences biologiquement défavorables, ne puisse pas quitter son domicile. Il était important de ne pas perdre la reine, car elle exerçait une action directe sur le mode et la durée de construction (R. DARCHEN, 1968).

L'écart entre la direction des rayons et celle des différents facteurs étudiés fut mesuré par l'angle des deux directions, situé à gauche de la direction de ces facteurs.

Le premier facteur étudié fut le champ magnétique terrestre. Pour cela on a utilisé la ruche en forme de tronc de cône. Comme le montrent les données du tableau 1 ou les photos de la Figure 1, les abeilles ont construit leurs rayons dans toutes les directions, c'est-à-dire sans être influencées par le champ magnétique terrestre. Notre résultat est en contradiction avec celui de OeHMKe (Martin et Lindauer, 1973). Nos expériences ne nous permettent pas de savoir si la différence de valeur de l'intensité magnétique de la terre entre la Grèce $(0,3$ gauss : K. Alexopoulos, 1959$)$ et l'Allemagne (0,5 gauss : MarTin et Lindauer, 1973) en est responsable. Nous ne pouvions pas non plus confirmer les dires de l'auteur cité, selon lesquels les premiers rayons d'un essaim dans un cylindre en carton ont la même direction que ceux de la colonie mère. On s'en rend compte en comparant les colonnes 2 et 3 du tableau 1.

Avec le même type de ruche on a testé l'action d'une lumière fixe. On a conservé le trou de vol au plancher, mais une petite ouverture latérale laissait entrer la lumière, et seulement elle, 24 heures par jour durant plusieurs semaines. On peut conclure des données du tableau 3 que la direction de la lumière n'influence pas la direction des rayons.

Dans une autre expérience fut testé le facteur "position du trou de vol ". La lumière solaire et l'air pénétraient ensemble par un trou de vol latéral dans la ruche en forme de tronc de cône. La figure 3 révèle que la position du trou de vol n'exerce pas elle non plus d'influence sur la direction des rayons, car elle variait d'une colonie à l'autre et d'un moment à l'autre pour la même colonie.

Nous avons étudié dans la ruche ellipsoïdale l'action de la forme du toit ou du volume intérieur du nid sur la direction des rayons. Les données du tableau 2 montrent que les abeilles ne sont pas non plus influencées par ce facteur dans la construction de leurs rayons. Les clichés de la figure 2 illustrent ces faits. 
Dans la ruche ellipsoïdale nous avons aussi utilisé le « faux col ». Dans ce cas la possibilité de communication entre les abeilles ainsi que la cohérence de la colonie sont tout d'abord maintenues. En outre l'existence de surfaces perpendiculaires à l'intérieur de la ruche est évitée systématiquement; parallèlement à cela cette barrière ne permet pas la construction d'un rayon qui traverse le toit dans toute sa longueur.

Lorsque les abeilles d'une colonie sont influencées par un facteur quelconque (par exemple le champ magnétique terrestre) dans l'orientation de leurs rayons à un moment donné, elles doivent coordonner les deux centres de construction, que la nouvelle disposition expérimentale fait presque toujours naître, et construire leurs rayons constamment selon la même direction. Mais les résultats représentés dans la figure $6 \mathrm{c}$ montrent, qu'une telle coordination n'existe nullement. Les résultats de cette expérience devraient prouver définitivement que les abeilles construisent leurs rayons selon les directions qui leur plaisent.

La figure 5 a montre le cas inattendu, ou les abeilles ont construit leurs rayons non seulement de bas en haut, mais aussi selon la forme d'un cylindre. Le phénomène s'est présenté dans 2 colonies que l'on avait forcées à construire de nouveaux rayons jusqu'à la mi-décembre. A cause des températures basses de cette saison les abeilles devaient rester près de la source de nourriture qui se trouvait sur le plancher de la ruche. Elles mirent ainsi en sûreté leur approvisionnement en candi.

Le cas représenté par la figure 5 a, dans lequel deux colonies ont chacune construit un rayon cylindrique, pourrait être la confirmation que le relief de la surface de base, sur lequel sont fixés les rayons, est le facteur décisif pour l'orientation des rayons. Ceci serait d'une très grande importance pour les abeilles elles-mêmes, parce qu'elles pourraient exploiter les cavités aux voûtes inégales qu'offre la nature, indépendamment de leur forme et de leur orientation et augmenteraient ainsi leurs chances de survie. 